\title{
Domain Decomposition Methodology with Robin Interface Matching Conditions for Solving Strongly Coupled Problems
}

\author{
François-Xavier Roux \\ ONERA, High performance Computing Unit, \\ 29 avenue de la Division Leclerc, 92320 CHATILLON, France \\ roux@onera.fr, \\ http://www.onera.fr
}

\begin{abstract}
In the case of strongly coupled problems like fluid-structure models in aero-elasticity or aero-thermo-mechanics, a standard solution methodology is based on so called Dirichlet-Neumann iterations. This means that, for instance, the velocity at the interface between the two media is imposed in the fluid, the solution of the fluid problem gives a pressure that is imposed at the boundary of the structure, and then the solution of the problem in the structure gives a new velocity to be imposed to the fluid. This method is not always stable, depending on the relative properties of the media, unless a suitable relaxation parameter is introduced. In order to enforce both velocity and pressure continuity at the interface, the matching conditions can be formulated, like in domain decomposition methods, in a mixed form. This means that the boundary conditions derived in one physical domain from the other one is of Robin type. With Robin boundary condition, an interface stiffness, in the case of velocity-pressure conditions, is introduced. The optimal choice for this stiffness can be proved to be, in the case of linear problems, the so called "Dirichlet-Neumann" operator of the opposite domain, this means for the discrete equations, the static condensation on the interface of the domain stiffness matrix. Of course, the static condensation cannot be performed in practice, since it is extremely expensive and that the resulting matrix is dense. But it can be approximated in several ways. The underlying general idea behind that methodology is the following: with Robin boundary conditions on the interface, a constitutive law is imposed on the boundary of each media that should optimally exactly represent the interaction with the other media.
\end{abstract}

Keywords: Domain decomposition, strongly coupled, fluid-structure coupling.

\section{Introduction}

Fluid-structure interaction for aero-elasticity of airplanes or for aero-thermomechanics of aeronautical engines involve strong coupling. That means that not only primal quantities, like temperature or velocities, must be continuous, but 
also dual quantities, like heat flux or pressure, must be balanced. The solution context then is mathematically similar to the one of domain decomposition for a single equation.

A very standard methodology for solving strongly coupled problem relies on alternate iterations on the various quantities at the interface between the domains until stagnation. When stagnation occurs, interface continuity requirements are fulfilled.

In the context of domain decomposition method, this methodology is called, for second order PDEs like steady heat equation or linear elasticity, DirichletNeumann iteration [1. In the second section of this paper standard convergence results for this method will be recalled. In the third section, Robin interface conditions will be introduced and will be proved to give a better stability to the method. General methodological approaches to design optimal Robin interface conditions will be mentioned in the concluding section.

\section{Dirichlet-Neumann Iterations}

\subsection{Model Problem}

For a sake of simplicity, we consider the steady heat transfer problem with two domains $\Omega_{1}$ and $\Omega_{2}$. Note $\Gamma_{3}=\partial \Omega_{1} \bigcap \partial \Omega_{2}$, the interface between both domains, like in Fig. 1]. Inside each domain, the steady heat equation must be solved.

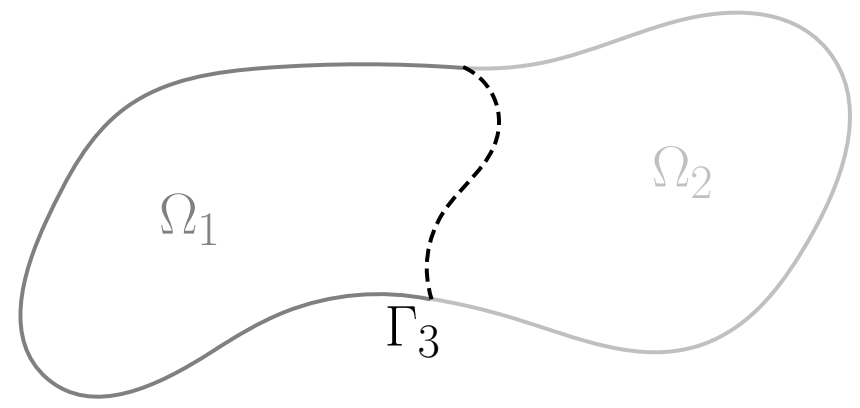

Fig. 1. Splitting in two domains

$$
\left\{\begin{array}{cc}
-k_{i} \Delta u_{i} & =f_{i} \quad \text { in } \Omega_{i} \\
u_{i} & =0 \text { on } \partial \Omega_{i} \backslash \Gamma_{3}
\end{array}\right.
$$

The Stokes formula gives the variational form of equation (1).

$$
\int_{\Omega_{i}} k_{i} \nabla u_{i} \nabla v_{i}=\int_{\Omega_{i}} f_{i} v_{i}+\int_{\Gamma_{3}} k_{i} \frac{\partial u_{i}}{\partial n_{i}} v_{i} \quad \forall v_{i} \in H_{0 \partial \Omega_{i} \backslash \Gamma_{3}}^{1}\left(\Omega_{i}\right)
$$

The interface coupling conditions are then continuity of the temperature:

$$
u_{1}-u_{2}=0 \text { on } \Gamma_{3}
$$

and equilibrium of heat flux: 


$$
k_{1} \frac{\partial u_{1}}{\partial n_{1}}=-k_{2} \frac{\partial u_{2}}{\partial n_{2}} \quad \text { on } \quad \Gamma_{3}
$$

The principle of Dirichlet-Neumann method is as follows. Solve the problem in $\Omega_{1}$, with prescribed values of $u_{1}$ on interface (Dirichlet problem). Compute the flux $k_{1} \frac{\partial u_{1}}{\partial n_{1}}$ on $\Gamma_{3}$. Solve the problem in $\Omega_{2}$ with balanced flux $k_{2} \frac{\partial u_{2}}{\partial n_{2}}=-k_{1} \frac{\partial u_{1}}{\partial n_{1}}$ on $\Gamma_{3}$ (Neumann problem). Take the trace on interface of the solution of Neumann problem in $\Omega_{2}$ and impose it as Dirichlet boundary condition in $\Omega_{1}, u_{1}=u_{2}$ on $\Gamma_{3}$. Restart iteration until stagnation.

This simple iterative process consists in enforcing alternatively the continuity of temperature and the equilibrium of flux at interface. It is clear that if the process converges it gives local solutions that satisfy both interface conditions of equations (3) and (4). Hence, the converged local solution fields satisfy the global coupled problem.

\subsection{Discretization}

Consider a discretization of equation (11) using finite element method. Each subdomain has its own mesh and the interface nodes are present in both meshes as in Fig. 2. Local stiffness matrices of the discrete problems in the two domains



Fig. 2. Two meshes with interface

can be written like in equation (5), using subscripts 1 and 2 for inner degrees of freedom of domains $\Omega_{1}$ and $\Omega_{2}$ and subscript 3 for degrees of freedom of interface $\Gamma_{3}$. Interface $\Gamma_{3}$ is present in both meshes. Hence, there are two interface blocks, one in each local matrix, noted with superscripts (1) and (2).

$$
K_{1}=\left[\begin{array}{ll}
K_{11} & K_{13} \\
K_{31} & K_{33}^{(1)}
\end{array}\right] \quad K_{2}=\left[\begin{array}{ll}
K_{22} & K_{23} \\
K_{32} & K_{33}^{(2)}
\end{array}\right]
$$


The discretization of variational formulation of equation (2) in domain $\Omega_{i}$ is:

$$
\left[\begin{array}{ll}
K_{i i} & K_{i 3} \\
K_{3 i} & K_{33}^{(i)}
\end{array}\right]\left[\begin{array}{c}
x_{i} \\
x_{3}^{(i)}
\end{array}\right]=\left[\begin{array}{l}
b_{i} \\
b_{3}^{(i)}+g_{3}^{(i)}
\end{array}\right]
$$

where $g_{3}^{(i)}$ is the vector representing the discretization of flux $k_{i} \frac{\partial u_{i}}{\partial n_{i}}$ on $\Gamma_{3}$.

For vectors that satisfy inner equation of (6]), there is an explicit relationship between the inner values and the interface values:

$$
x_{i}=K_{i i}^{-1} b_{i}-K_{i i}^{-1} K_{i 3} x_{3}^{(i)}
$$

From explicit relation between inner and interface values in equation (77) and thanks to the representation of discrete flux in equation (6), the standard following relation linking the trace and the flux of a vector satisfying inner equation can be derived:

$$
\begin{aligned}
g_{3}^{(i)} & =K_{3 i} x_{i}+K_{33}^{(i)} x_{3}^{(i)}-b_{3}^{(i)} \\
& =K_{3 i}\left(K_{i i}^{-1} b_{i}-K_{i i}^{-1} K_{i 3} x_{3}^{(i)}\right)+K_{33}^{(i)} x_{3}^{(i)}-b_{3}^{(i)} \\
& =\left(K_{33}^{(i)}-K_{3 i} K_{i i}^{-1} K_{i 3}\right) x_{3}^{(i)}-\left(b_{3}^{(i)}-K_{3 i} K_{i i}^{-1} b_{i}\right) \\
& =S^{(i)} x_{3}^{(i)}-c_{3}^{(i)}
\end{aligned}
$$

Matrix $S^{(i)}=K_{33}^{(i)}-K_{3 i} K_{i i}^{-1} K_{i 3}$ is the Schur complement matrix. It is the discretization of the so called Dirichlet to Neumann mapping that defines the bi-continuous one to one correspondence between the trace and the flux on the boundary of a field that satisfies the steady heat equation inside the domain. It is symmetric positive definite if the $K_{i}$ matrix is symmetric positive definite. The same holds, of course, for any other second order coercive PDE.

For the Dirichlet problem in domain $\Omega_{1}$, only test functions with null trace on $\Gamma_{3}$ have to be considered, so the discrete form of this problem is, for a prescribed trace $x_{3}$ on interface:

$$
\left\{\begin{array}{l}
K_{11} x_{1}+K_{13} x_{3}^{(1)}=b_{1} \\
x_{3}^{(1)}=x_{3}
\end{array}\right.
$$

The discrete flux associated with the solution of equation (9) can be recovered from equation (6), as already done to define Schur complement in equation (8):

$$
g_{3}^{(1)}=K_{31} x_{1}+K_{33}^{(1)} x_{3}^{(1)}-b_{3}^{(1)}
$$

Equation (6) gives directly the discrete formulation of Neumann problem in domain $\Omega_{2}$ for a prescribed value $g_{3}^{(2)}$ of flux on $\Gamma_{3}$ :

$$
\left[\begin{array}{ll}
K_{22} & K_{23} \\
K_{32} & K_{33}^{(2)}
\end{array}\right]\left[\begin{array}{c}
x_{2} \\
x_{3}^{(2)}
\end{array}\right]=\left[\begin{array}{l}
b_{2} \\
b_{3}^{(2)}+g_{3}^{(2)}
\end{array}\right]
$$

Finally, continuity relations (3) and (4) have the following discrete counterparts:

$$
x_{3}^{(1)}=x_{3}^{(2)}
$$




$$
g_{3}^{(1)}=-g_{3}^{(2)}
$$

If local solution vectors satisfy the continuity condition (12), then, thanks to equations (9) and (11), the global vector $x$, with $x_{3}=x_{3}^{(1)}=x_{3}^{(2)}$ satisfy inner equations in domains $\Omega_{1}$ and $\Omega_{2}$ :

$$
\left\{\begin{array}{l}
K_{11} x_{1}+K_{13} x_{3}=b_{1} \\
K_{22} x_{2}+K_{23} x_{3}=b_{2}
\end{array}\right.
$$

Equilibrium condition (13), combined with local equations (10) and (11), gives the following interface equation:

$$
\begin{gathered}
K_{31} x_{1}+K_{33}^{(1)} x_{3}-b_{3}^{(1)}=-\left(K_{32} x_{2}+K_{33}^{(2)} x_{3}-b_{3}^{(2)}\right) \Leftrightarrow \\
K_{31} x_{1}+K_{32} x_{2}+\left(K_{33}^{(1)}+K_{33}^{(2)}\right) x_{3}=b_{3}^{(1)}+b_{3}^{(2)}
\end{gathered} \Leftrightarrow
$$

Inner equations (14) and interface equation (15) mean that local solution vectors satisfying both the continuity condition (12) and the equilibrium condition (13) define a global vector solution of the assembled global problem:

$$
\left[\begin{array}{ccc}
K_{11} & 0 & K_{13} \\
0 & K_{22} & K_{23} \\
K_{31} & K_{32} & K_{33}
\end{array}\right]\left[\begin{array}{l}
x_{1} \\
x_{2} \\
x_{3}
\end{array}\right]=\left[\begin{array}{l}
b_{1} \\
b_{2} \\
b_{3}
\end{array}\right]
$$

where $K_{33}=K_{33}^{(1)}+K_{33}^{(2)}$ and $b_{3}=b_{3}^{(1)}+b_{3}^{(2)}$.

Of course, equation (16) is the standard discretization of the global coupled problem, that is, in the present case, the steady heat equation on the complete domain.

Performing elimination of inner unknowns as already done to define Schur complement in equation (8) in the global coupled system (16) leads to a coupled condensed problem on $\Gamma_{3}$ :

$$
\left(K_{33}-K_{31} K_{11}^{-1} K_{13}-K_{32} K_{22}^{-1} K_{23}\right) x_{3}=b_{3}-K_{31} K_{11}^{-1} b_{1}-K_{32} K_{22}^{-1} b_{2}
$$

The complete Schur complement $S=K_{33}-K_{31} K_{11}^{-1} K_{13}-K_{32} K_{22}^{-1} K_{23}$ is the sum of the two local ones:

$$
S=K_{33}^{(1)}+K_{33}^{(2)}-K_{31} K_{11}^{-1} K_{13}-K_{32} K_{22}^{-1} K_{23}=S^{(1)}+S^{(2)}
$$

In the same way, the right hand side of equation (17) is the sum of two local contributions:

$$
c_{3}=b_{3}^{(1)}+b_{3}^{(2)}-K_{31} K_{11}^{-1} b_{1}-K_{32} K_{22}^{-1} b_{2}=c_{3}^{(1)}+c_{3}^{(2)}
$$




\subsection{Discrete Dirichlet-Neumann Iteration}

Iteration $p$ of Dirichlet-Neumann iteration consists in the following steps.

1. Given $x_{3}^{p}$, solve the Dirichlet problem in domain $\Omega_{1}$ :

$$
\left\{\begin{array}{l}
K_{11} x_{1}^{p}+K_{13} x_{3}^{(1) p}=b_{1} \\
x_{3}^{(1) p}=x_{3}^{p}
\end{array}\right.
$$

2. Compute interface flux:

$$
g_{3}^{(1) p}=K_{31} x_{1}^{p}+K_{33}^{(1)} x_{3}^{(1) p}-b_{3}^{(1)}
$$

3. Solve Neumann problem in domain $\Omega_{2}$ :

$$
\left[\begin{array}{ll}
K_{22} & K_{23} \\
K_{32} & K_{33}^{(2)}
\end{array}\right]\left[\begin{array}{c}
x_{2}^{p} \\
x_{3}^{(2) p}
\end{array}\right]=\left[\begin{array}{l}
b_{i} \\
b_{3}^{(2)}-g_{3}^{(1) p}
\end{array}\right]
$$

4. Derive new value of interface trace:

$$
x_{3}^{p+1}=x_{3}^{(2) p}
$$

In reality, only interface values matter in the iteration, since inner values of $x$ can be derived from interface ones, thanks to the inner equations (14). More precisely, thanks to the relationship between $x_{3}$ and $g_{3}$ via the Schur complement as stated in equation (8), the Dirichlet-Neumann iteration can be redefined only in terms of values of $x_{3}$ and $g_{3}$.

1. Given $x_{3}^{p}$, solve the Dirichlet problem in domain $\Omega_{1}$ and compute interface flux:

$$
g_{3}^{(1) p}=S^{(1)} x_{3}^{p}-c_{3}^{(1)}
$$

2. Derive new value of interface trace from solution of Neumann problem in domain $\Omega_{2}$ :

$$
S^{(2)} x_{3}^{p+1}=c_{3}^{(2)}-g_{3}^{(1) p}
$$

Finally, $x_{3}^{p}$ and $x_{3}^{p+1}$ satisfy the following relation:

$$
S^{(2)} x_{3}^{p+1}=-S^{(1)} x_{3}^{p}+c_{3}^{(1)}+c_{3}^{(2)}
$$

Equation (26) shows that the Dirichlet-Neumann iteration is a relaxation iteration for the coupled global problem whose condense form stated in equation (17) can be written:

$$
\left(S^{(1)}+S^{(2)}\right) x_{3}=c_{3}^{(1)}+c_{3}^{(2)}
$$

The iteration matrix for the error of this method is derived from equation (26):

$$
\begin{aligned}
x_{3}^{p+1} & =-S^{(2)-1} S^{(1)} x_{3}^{p}+S^{(2)-1} c_{3}=-S^{(2)-1} S^{(1)} x_{3}^{p}+S^{(2)-1} S x_{3} \\
& =-S^{(2)-1}\left(S-S^{(2)}\right) x_{3}^{p}+S^{(2)-1} S x_{3}=-S^{(2)-1} S x_{3}^{p}+x_{3}^{p}+S^{(2)-1} S x_{3}
\end{aligned}
$$

and so:

$$
\left.x_{3}^{p+1}-x_{3}=\left(I-S^{(2)-1} S\right)\left(x_{3}^{p}-x_{3}\right)=-S^{(2)-1} S^{(1)}\right)\left(x_{3}^{p}-x_{3}\right)
$$


Equation (29) shows that the method converges if and only if $\left\|S^{(2)-1} S^{(1)}\right\|<1$. This means that $S^{(2)}$ must be, in some sense, larger than $S^{(1)}$. Suppose the two domains are geometrically symmetric, with the same thermal conductivity, then the $S^{(1)}$ and $S^{(2)}$ matrices are identical. If the thermal conductivity is different from one domain to the other, the ratio between the two matrices is equal to the ratio between the thermal conductivity of both domains. This means that the Dirichlet-Neumann iteration will converge if the Dirichlet problem is solved in the domain with lower conductivity and the Neumann problem in the domain with the higher conductivity. The physical interpretation is that the domain with the higher conductivity receives the heat flux on the interface from the domain with the lower conductivity and in return imposes its temperature on the interface.

Convergence can be obtained by introducing a suitable over-relaxation parameter $\beta$ :

$$
x_{3}^{p+1}=(1-\beta) x_{3}^{p}+\beta x_{3}^{p+\frac{1}{2}}
$$

where $x_{3}^{p+\frac{1}{2}}$ is given by the simple Dirichlet-Neumann iteration like in equation (26). This leads to a new iteration matrix for the error:

$$
x_{3}^{p+1}-x_{3}=\left(I-\beta S^{(2)-1} S\right)\left(x_{3}^{p}-x_{3}\right)
$$

Since $S^{(2)}$ and $S$ are symmetric positive definite, $\left\|I-\beta S^{(2)-1} S\right\|<1$ if $\beta$ is small enough. Unfortunately, if the Neumann problem is not solved on the right side, $\beta$ may have to be chosen very small and, in such a case, the convergence is very slow. Furthermore, it may be very difficult to find the right value of $\beta$ if the materials are heterogeneous on both sides of the interface.

\section{Robin Interface Condition}

\subsection{Robin Boundary Condition}

The continuous Robin boundary condition for the steady heat equation in domain $\Omega_{i}$ takes the following form:

$$
k_{i} \frac{\partial u_{i}}{\partial n_{i}}+\alpha_{i} u_{i}=w_{i} \quad \text { on } \Gamma_{3}
$$

Introducing the Robin boundary condition (32) in variational formula (2) leads to the following new variational form for the steady heat equation in domain $\Omega_{i}$ :

$$
\int_{\Omega_{i}} k_{i} \nabla u_{i} \nabla v_{i}+\int_{\Gamma_{3}} \alpha_{i} u_{i} v_{i}=\int_{\Omega_{i}} f_{i} v_{i}+\int_{\Gamma_{3}} w_{i} v_{i} \quad \forall v_{i} \in H_{0 \partial \Omega_{i} \backslash \Gamma_{3}}^{1}\left(\Omega_{i}\right)
$$

The discretization of variational formulation of equation (33) in domain $\Omega_{i}$ is:

$$
\left[\begin{array}{cc}
K_{i i} & K_{i 3} \\
K_{3 i} & K_{33}^{(i)}+A_{33}^{(i)}
\end{array}\right]\left[\begin{array}{c}
x_{i} \\
x_{3}^{(i)}
\end{array}\right]=\left[\begin{array}{l}
b_{i} \\
b_{3}^{(i)}+\tilde{g}_{3}^{(i)}
\end{array}\right]
$$


where $\tilde{g}_{3}^{(i)}$ is the vector representing the discretization of augmented flux $k_{i} \frac{\partial u_{i}}{\partial n_{i}}+$ $\alpha_{i} u_{i}$ on $\Gamma_{3}$.

Elimination of inner unknowns in equation (34) gives the following relation between the trace of the solution of the Robin problem and the discretization of augmented flux on $\Gamma_{3}$ :

$$
\begin{aligned}
\tilde{g}_{3}^{(i)} & =K_{3 i} x_{i}+\left(K_{33}^{(i)}+A_{33}^{(i)}\right) x_{3}^{(i)}-b_{3}^{(i)} \\
& =K_{3 i}\left(K_{i i}^{-1} b_{i}-K_{i i}^{-1} K_{i 3} x_{3}^{(i)}\right)+\left(K_{33}^{(i)}+A_{33}^{(i)}\right) x_{3}^{(i)}-b_{3}^{(i)} \\
& =\left(K_{33}^{(i)}+A_{33}^{(i)}-K_{3 i} K_{i i}^{-1} K_{i 3}\right) x_{3}^{(i)}-\left(b_{3}^{(i)}-K_{3 i} K_{i i}^{-1} b_{i}\right) \\
& =\left(S^{(i)}+A_{33}^{(i)}\right) x_{3}^{(i)}-c_{3}^{(i)}
\end{aligned}
$$

This means that augmented system (34) gives an augmented Schur complement with the same augmentation matrix $A_{33}^{(i)}$.

The relation between the discretization of the flux $k_{i} \frac{\partial u_{i}}{\partial n_{i}}$ and the augmented flux is $k_{i} \frac{\partial u_{i}}{\partial n_{i}}+\alpha_{i} u_{i}$ given by:

$$
\begin{aligned}
g_{3}^{(i)} & =\tilde{g}_{3}^{(i)}-A_{33}^{(i)} x_{3}^{(i)} \\
& =\left(S^{(i)}\right) x_{3}^{(i)}-c_{3}^{(i)}
\end{aligned}
$$

\subsection{Dirichlet-Robin Method}

The Dirichlet-Robin method consists in enforcing the equilibrium of flux between domain $\Omega_{1}$ and $\Omega_{2}$ via a mixed condition, leading to a Robin problem in $\Omega_{2}$ :

$$
k_{2} \frac{\partial u_{2}}{\partial n_{2}}+\alpha_{2} u_{2}=-k_{1} \frac{\partial u_{1}}{\partial n_{1}}+\alpha_{2} u_{1} \quad \text { on } \Gamma_{3}
$$

The discrete Dirichlet-Robin can be directly written in term of interface unknowns:

1. Given $x_{3}^{p}$, solve the Dirichlet problem in domain $\Omega_{1}$ and compute interface flux:

$$
g_{3}^{(1) p}=S^{(1)} x_{3}^{p}-c_{3}^{(1)}
$$

2. Derive new value of interface trace from solution of Robin problem in domain $\Omega_{2}$ :

$$
\left(S^{(2)}+A_{33}^{(2)}\right) x_{3}^{p+1}=c_{3}^{(2)}-g_{3}^{(1) p}+A_{33}^{(2)} x_{3}^{p}
$$

Finally, $x_{3}^{p}$ and $x_{3}^{p+1}$ satisfy the following relation:

$$
\left(S^{(2)}+A_{33}^{(2)}\right) x_{3}^{p+1}=\left(-S^{(1)}+A_{33}^{(2)}\right) x_{3}^{p}+c_{3}^{(1)}+c_{3}^{(2)}
$$

Since the solution of coupled problem (17) satisfies the same equation as (40):

$$
\left(S^{(1)}+S^{(2)}+A_{33}^{(2)}-A_{33}^{(2)}\right) x_{3}=c_{3}^{(1)}+c_{3}^{(2)}
$$

the iteration matrix for the error of this method is given by the following equation:

$$
x_{3}^{p+1}-x_{3}=-\left(S^{(2)}+A_{33}^{(2)}\right)^{-1}\left(S^{(1)}-A_{33}^{(2)}\right)\left(x_{3}^{p}-x_{3}\right)
$$


The method converges if and only if $\left\|\left(S^{(2)}+A_{33}^{(2)}\right)^{-1}\left(S^{(1)}-A_{33}^{(2)}\right)\right\|<1$. It can be the case, even though $S^{(2)}$ is not larger than $S^{(1)}$, provided that the augmentation matrix $A_{33}^{(2)}$ is close to the Shur complement of domain $\Omega_{1}, S^{(1)}$. If $A_{33}^{(2)}=S^{(1)}$, the method converges in only one iteration: it is a direct method.

This property can be also derived from the elimination of inner unknowns of domain $\Omega_{1}$ in global system (16), that reduces the system as follows in $\Omega_{2}$ :

$$
\left[\begin{array}{cc}
K_{22} & K_{23} \\
K_{32} & K_{33}-K_{31} K_{11}^{-1} K_{13}
\end{array}\right]\left[\begin{array}{l}
x_{2} \\
x_{3}
\end{array}\right]=\left[\begin{array}{l}
b_{2} \\
b_{3}-K_{31} K_{11}^{-1} b_{1}
\end{array}\right]
$$

This system can be rewritten:

$$
\left[\begin{array}{cc}
K_{22} & K_{23} \\
K_{32} & K_{33}^{(2)}+S^{(1)}
\end{array}\right]\left[\begin{array}{l}
x_{2} \\
x_{3}
\end{array}\right]=\left[\begin{array}{l}
b_{2} \\
b_{3}^{(2)}+c_{3}^{(1)}
\end{array}\right]
$$

Equation (44) means that the restriction in $\Omega_{2}$ of the solution of the global coupled problem is solution of a generalized local Robin problem, where the operator in the Robin condition is the Schur complement of domain $\Omega_{1}$.

This is hardly a surprise on a physical point of view. The Schur complement is the discretization of Dirichlet-Neumann mapping. If a temperature and a flux satisfy the relation given by equation (8), they are respectively the trace and the flux of solution of local problem (6).

A generalized Robin boundary condition sets a constitutive law for the interface. If the constitutive law on interface of domain $\Omega_{2}$ is such that the trace and the flux of the local problem in domain $\Omega_{2}$ with the corresponding Robin boundary condition are the trace and flux of the solution of the local problem in domain $\Omega_{1}$, it entails that the solution of Robin problem in $\Omega_{2}$ is the restriction of the global coupled problem. The optimal generalized Robin condition forces the boundary of a domain to behave in the same way as the boundary of the neighboring domain.

\subsection{Robin-Robin Method}

The Dirichlet problem in domain $\Omega_{1}$ can be also replaced by a Robin problem similar to (40). It can be proved that the iteration matrix for the error of the Robin-Robin method is equal to:

$$
\left(S^{(2)}+A_{33}^{(2)}\right)^{-1}\left(S^{(1)}-A_{33}^{(2)}\right)\left(S^{(1)}+A_{33}^{(1)}\right)^{-1}\left(S^{(2)}-A_{33}^{(1)}\right)
$$

Equation (45) means that performing a Robin-Robin iteration is equivalent to performing alternatively a Dirichlet-Robin and a Robin-Dirichlet iteration.

\section{Conclusion}

The coupling algorithms based on Robin interface conditions have many interesting features. First, Robin boundary condition can always be formulated in such a 
way that associated local problem is always well posed, even though the Neumann problem is not. Secondly, it is not necessary to impose the Robin condition on the "right" side to get convergence. It can even be imposed on both sides.

In practice however, it is not possible to compute the exact Schur complement that defines the optimal Robin interface condition, for several reasons. Its computation is very expensive and it is a dense operator that couples all interface unknowns. At continuous level, the Dirichlet-Neumann mapping is not local. Furthermore, it can be computed only for linear problems with adequate variational formulation.

Nevertheless, there are several approximation methods for the DirichletNeumann mapping. It can be done by defining a local approximation of the continuous operator, like in the methods to set up approximate absorbing conditions for wave equation 2, and by discretizing it. The analysis can also be performed directly on the discrete operator.

Any physical approach to set up the constitutive law associated with the optimal Dirichlet-Neumann mapping can be valuable as well.

Also, purely algebraic approaches, like the ones developed in [3] can also be used to compute a sparse approximation of the Schur complement. This methodology has already been successfully used for various coupled fluid-structure problems.

\section{References}

1. Funaro, D., Quarteroni, A., Zanolli, P.: An Iterative Procedure with Interface Relaxation for Domain Decomposition Methods. SIAM J. Numer. Anal. 25(6), 1213-1236 (1998)

2. Gander, M.J., Halpern, L., Nataf, F.: Optimal Schwarz Waveform Relaxation for the One Dimensional Wave Equation. SIAM J. Numer. Anal. 41, 1643-1681 (2003)

3. Magoules, F., Roux, F.-X., Series, L.: Algebraic Approximation of Dirichlet-toNeumann Maps for the Equations of Linear Elasticity. Computer Methods in Applied Mechanics and Engineering 195(29-32), 3742-3759 (2006) 